\title{
RNA sequencing identifies gene expression profile changes associated with $\beta$-estradiol treatment in U2OS osteosarcoma cells
}

This article was published in the following Dove Press journal:

OncoTargets and Therapy

II July 2017

Number of times this article has been viewed

\section{Bin Chen \\ Zude Liu \\ Jidong Zhang \\ Hantao Wang \\ Bo Yu}

Department of Orthopedic Surgery, Renji Hospital, School of Medicine, Shanghai Jiao Tong University, Shanghai, People's Republic of China
Correspondence: Zude Liu; Jidong Zhang Department of Orthopedic Surgery, Renji Hospital, School of Medicine, Shanghai Jiao Tong University, No I60, Pujian Road, Shanghai 200I27, People's Republic of China

Tel +86 2I 68383705

Fax +86 2I 58395057

Email zudeliu2016vip@I63.com; zhangjidong@renji.com
Abstract: This study was conducted to identify gene expression profile changes associated with $\beta$-estradiol (E2) treatment in U2OS osteosarcoma cells by high-throughput RNA sequencing (RNA-seq). Two U2OS cell samples treated with E2 $(15 \mu \mathrm{mol} / \mathrm{L})$ and two untreated control U2OS cell samples were subjected to RNA-seq. Differentially expressed genes (DEGs) between the groups were identified, and main biological process enrichment was performed using gene ontology (GO) analysis. A protein-protein interaction (PPI) network was constructed using Cytoscape based on the Human Protein Reference Database. Finally, NFKB1 expression was confirmed by quantitative real-time polymerase chain reaction (qRT-PCR). The map ratios of the four sequenced samples were $>65 \%$. In total, 128 upregulated and 92 downregulated DEGs were identified in E2 samples. After GO enrichment, the downregulated DEGs, such as $A K T 1$, were found to be mainly enriched in cell cycle processes, whereas the upregulated DEGs, such as NFKB1, were involved in the regulation of gene expression. Moreover, AKT1 (degree =117) and NFKB1 (degree $=72$ ) were key nodes with the highest degrees in the PPI network. Similarly, the results of qRT-PCR confirmed that E2 upregulated $N F K B 1$ expression. The results suggest that E2 upregulates the expression of $N F K B 1, A T F 7 I P$, and $H D A C 5$, all of which are involved in the regulation of gene expression and transcription, but downregulates that of TCF7L2, ALCAM, and $A K T$, which are involved in Wnt receptor signaling through $\beta$-catenin and morphogenesis in U2OS osteosarcoma cells.

Keywords: differentially expressed genes, Wnt receptor signaling, $\beta$-catenin, protein-protein interaction network

\section{Introduction}

$17 \beta$-Estradiol (E2) is a primary sex hormone in human beings that is essential for the development and maintenance of female reproductive organs. ${ }^{1}$ However, it also has important effects on many other tissues, such as bone, ${ }^{2}$ liver, ${ }^{3}$ and brain. ${ }^{4} \mathrm{E} 2$ is mainly produced by the granulosa cells of ovaries in women, ${ }^{5}$ but it can also be produced by the testes in men. ${ }^{6,7}$ E2 has been used for treating menopausal syndrome ${ }^{8}$ and preventing osteoporosis in postmenopausal women. ${ }^{9}$ In addition, it has been reported that older men with total E2 deficiency are more likely to be osteoporotic. ${ }^{10}$ E2 has also been implicated in cancer progression. ${ }^{11,12}$ Recently, Tchafa et $\mathrm{al}^{13}$ have found that E2 promotes the cellular invasion and proliferation of breast cancer cells. Gunter et $\mathrm{al}^{12}$ have reported that endogenous E2 levels are positively associated with the risk of colorectal cancer.

Osteosarcoma is the most common primary malignancy of bone and exhibits a high risk of metastasis and poor prognosis. ${ }^{14,15}$ Although E2 is known to play a critical role 
in osteosarcoma, its effects in this disease are controversial. E2 can inhibit purine metabolic and biosynthetic pathways in human osteosarcoma cells to achieve an antagonistic effect on cell proliferation. ${ }^{16}$ Previous studies have also shown that E2 protects against cell death in estrogen receptor- $\alpha$ and - $\beta$-expressing human U2OS osteosarcoma cells. ${ }^{17,18}$ Furthermore, 2-methoxyestradiol, a mammalian metabolite of E2, has been reported to induce cell cycle arrest and osteosarcoma cell apoptosis. ${ }^{19}$ Therefore, defining the molecular mechanism(s) of E2 actions in osteosarcoma cells is necessary. In the present study, high-throughput RNA sequencing (RNA-seq) and bioinformatics methods were used to identify changes in the gene expression profile that are associated with E2 treatment of U2OS osteosarcoma cells.

\section{Materials and methods}

\section{Cell lines and culture conditions}

Human U2OS osteosarcoma cells were purchased from Cell Bank of Type Culture Collection of the Chinese Academy of Sciences (Shanghai, People's Republic of China). Cells were maintained in phenol red-free Roswell Park Memorial Institute (RPMI)-1640 medium supplemented with 10\% fetal bovine serum and $1 \%$ penicillin-streptomycin (Thermo Fisher Scientific, Waltham, MA, USA) in a humidified atmosphere of $95 \%$ air and $5 \% \mathrm{CO}_{2}$ at $37^{\circ} \mathrm{C}$. Before beginning treatments, cells were washed twice with phosphate-buffered saline (PBS) to remove residual serum and were grown in serum-free RPMI-1640 medium for 24 h. Subsequently, E2 (15 $\mu \mathrm{mol} / \mathrm{L}$, dissolved in dimethyl sulfoxide [DMSO]) was added to the medium, and an equal volume of DMSO was added to the control U2OS cell medium. Two U2OS cell samples (14710C-3 and 14710C-4) treated with E2 and two untreated control U2OS samples (14710C-1 and 14710C-2) were subjected to RNA-seq.

\section{RNA extraction and sequencing}

After an incubation period of $48 \mathrm{~h}$, cells were washed twice with PBS and harvested. Total RNA was isolated from cultured cells using TRIzol ${ }^{\circledR}$ Reagent (Thermo Fisher Scientific) according to the manufacturer's instruction. RNA quality and quantity were assessed by an Agilent 2100 Bioanalyzer (Agilent Technologies, Santa Clara, CA, USA). cDNA libraries were constructed using an NEBNext ${ }^{\circledR}$ Ultra $^{\text {TM }}$ RNA Library Prep Kit (Illumina, Shanghai, People's Republic of China) following the manufacturer's instruction. Subsequently, libraries were sequenced on Illumina HiSeq 2000 at Beijing Berry Genomics Co., Ltd. Sequenced reads were generated by base calling using the Illumina standard pipeline. Paired-end
RNA-seq data were generated with a read length of $100 \mathrm{bp}$. The raw sequencing data have been uploaded to the National Center for Biotechnology Information database under the BioProject accession no SRP101761.

\section{Alignment of sequenced reads}

Raw reads were first filtered to obtain clean reads using FASTX-Toolkit (http://hannonlab.cshl.edu/fastx toolkit/). ${ }^{20}$ High-quality reads were mapped to the human reference genome hg19 using TopHat (version 2.0.12) software ${ }^{21}$ with default parameters. Alignment was independently performed for reads from each sample, and reads mapping to more than three genomic sites were discarded.

\section{Differentially expressed genes (DEGs) screening}

Based on the value of reads per kilobase per million mapped reads, gene expression levels were determined using Cufflinks software (version 2.21). ${ }^{22}$ Subsequently, the Cuffdiff program ${ }^{23}$ of Cufflinks was used to identify DEGs. Only the genes with $\mid \log ($ fold change $) \mid>1$ and $P<0.01$ were considered as DEGs.

\section{Gene ontology (GO) enrichment analysis}

Database for Annotation, Visualization and Integrated Discovery (DAVID) ${ }^{24}$ is an online tool used for functional annotation of genes. A GO functional enrichment analysis of DEGs was performed using the DAVID. $P<0.05$ was chosen as the cutoff criterion.

\section{Construction of protein-protein interaction (PPI) network}

The Human Protein Reference Database (http://www.hprd. org/ $)^{25}$ is a database for experimentally derived information about the human proteome, including that on PPIs, post-translational modifications, and tissue expression. DEG-encoding proteins were mapped to the Human Protein Reference Database to search for interaction relationships. The PPI network was visualized using Cytoscape. ${ }^{26}$ Finally, the hub nodes with a high degree of connectivity ${ }^{27}$ in the PPI network were also identified.

\section{NFKBI expression levels using quantitative real-time polymerase chain reaction ( $q R T-P C R$ )}

To confirm RNA-seq results, NFKB1 and AKT1 expression levels were detected using qRT-PCR. Total RNA was extracted using TRIzol Reagent following the manufacturer's 
instructions (TaKaRa, Dalian, People's Republic of China). Subsequently, the first-strand cDNA was prepared from total lens RNA using a TaKaRa PrimeScript II First Strand cDNA Synthesis Kit (RR036A-1; TaKaRa) according to the manufacturer's instructions. Glyceraldehyde-3-phosphate dehydrogenase was used as a control. The primers used for NFKB1, $A K T 1$, and glyceraldehyde-3-phosphate dehydrogenase were based on the rat sequences: $5^{\prime}$-AACAGCAGATGGCCCATA CC-3' (forward), 5'-AACCTTTGCTGGTCCCACAT-3' (reverse); 5'-GCCTGTCAGCTGGTGCAT-3' (forward), 5'-CCGCCAGGTCTTGATGTACT-3' (reverse); and 5'-CA GTGCCAGCCTCGTCTCAT-3' (forward), 5'-AGG GGCCATCCACAGTCTTC-3' (reverse), respectively.

\section{Statistical analysis}

Differences between the two treatment groups were analyzed using unpaired Student's $t$-test. Data analysis was completed using SPSS 22.0 (IBM Corporation, Armonk, NY, USA). $P<0.05$ was considered to indicate statistically significant difference.

\section{Results}

\section{Sequence alignment}

The results of TopHat alignment of clean reads with the human reference genome are shown in Table 1. In total, $9,485,360(68.63 \%)$ and 7,843,445 (65.76\%) of clean reads were mapped to the human reference genome for the two E2-treated U2OS cell samples; 8,129,145 (69.71\%) and $8,531,536(69.07 \%)$ cleans reads were mapped for the two control samples.

\section{DEGs and GO enrichment analysis}

In total, 220 genes, including 128 upregulated and 92 downregulated genes, were identified as being significantly differently expressed between E2-treated U2OS osteosarcoma cells and the controls. According to the GO enrichment analysis, the top five GO terms of upregulated DEGs NFKB1, ATF7IP, $H D A C 5, M E N 1$, and EPC1 were significantly related to the

Table I Summary of clean reads alignment to the reference genome

\begin{tabular}{|c|c|c|c|c|}
\hline Sample title & Treatment & Clean reads & $\begin{array}{l}\text { Mapped } \\
\text { reads }\end{array}$ & $\begin{array}{l}\text { Map } \\
\text { ratio (\%) }\end{array}$ \\
\hline I47I0C-I & Control & 11660638 & 8129145 & 69.71 \\
\hline $147 \mid 0 C-2$ & Control & $|235| 70 \mid$ & 8531536 & 69.07 \\
\hline $147 \mid 0 C-3$ & E2 & $|38205| 4$ & 9485360 & 68.63 \\
\hline $147 \mid 0 C-4$ & E2 & | |92758| & 7843445 & 65.76 \\
\hline
\end{tabular}

Abbreviation: E2, estradiol. regulation of gene expression and transcription (Table 2). Meanwhile, the top five GO terms of downregulated DEGs included Wnt receptor signaling through $\beta$-catenin involving $R A R G, T B L 1 X$, and $T C F 7 L 2$; axonogenesis involving $A L C A M, N R P 1$, and SLC26A6; and cell cycle processes involving AKT1, DSN1, and POLD1 (Table 3).

\section{Construction of the PPI network}

In total, 1,185 nodes, including 91 upregulated DEGs, 55 downregulated DEGs, and 1,064 non-DEGs, were present in the PPI network (Figure 1). The top five DEGs with the highest degree of connectivity in the network were AKT1 (117), NFKB1 (72), ATF7IP (64), NCOA3 (45), and HDAC5 (36).

\section{Differences in NFKBI and AKTI expression levels}

As shown in Figure 2A, NFKB1 expression levels significantly increased when U2OS osteosarcoma cells were treated with E2 $(P=0.002)$, which confirmed the reliability of the bioinformatics method. Although $A K T 1$ expression levels were increased when U2OS osteosarcoma cells were treated with $\mathrm{E} 2$, they were not significantly different $(P>0.05$; Figure 2B).

\section{Discussion}

In the current study, RNA-seq was used to explore changes in the gene expression profile that are associated with E2 treatment of U2OS osteosarcoma cells. We found that E2 treatment induced the upregulation of genes related to the regulation of gene expression and transcription (eg, $N F K B 1$, $A T F 7 I P$, and $H D A C 5$ ) and downregulation of those involved in Wnt receptor signaling through $\beta$-catenin and morphogenesis (eg, TCF7L2, ALCAM, NRP1, SLC26A6, and AKT).

Our results demonstrated that $N F K B 1$ was mainly enriched in the regulation of gene expression and transcription. $N F K B 1$ belongs to the $N F-\kappa B$ family, which contains a group of proteins involved in carcinogenesis, immune response, cell adhesion, proliferation, angiogenesis, and apoptosis. ${ }^{28} \mathrm{NF}-\kappa \mathrm{B}$ is a transcription factor that participates in the regulation of viral and cellular genes. ${ }^{29}$ Constitutive $N F-\kappa B$ activation has been observed in $67 \%$ of colorectal cancer cell lines and promoted tumor growth. ${ }^{30} \mathrm{NFKB} 2$, another member of the $N F-\kappa B$ family, can stimulate cell proliferation in U2OS osteosarcoma cells. ${ }^{31}$ Furthermore, several studies have shown the relationship between $N F K B 1$ and tumors. For example, a functional insertion/deletion polymorphism in the promoter region of $N F K B 1$ increases the risk of 
Table 2 The top five GO terms of upregulated DEGs

\begin{tabular}{|c|c|c|c|c|}
\hline Category & Term & Description & P-value & Genes \\
\hline BP & GO:0010628 & Positive regulation of gene expression & 0.002272 & NFKBI, ATF7IP, HDAC5, etc \\
\hline BP & GO:0010629 & Negative regulation of gene expression & 0.002581 & NFKBI, ATF7IP, HDAC5, etc \\
\hline $\mathrm{BP}$ & GO:0016568 & Chromatin modification & 0.002959 & HDAC5, MENI, EPCI, etc \\
\hline BP & GO:0045944 & Positive regulation of transcription from RNA polymerase II promoter & 0.004248 & NFKBI, HDAC5, MENI, etc \\
\hline $\mathrm{BP}$ & GO:00I648I & Negative regulation of transcription & 0.004572 & NFKBI, ATF7IP, HDAC5, etc \\
\hline
\end{tabular}

Abbreviations: BP, biological process; GO, gene ontology; DEGs, differentially expressed genes.

nasopharyngeal carcinoma. ${ }^{32}$ Riemann et al ${ }^{33}$ discovered that the NFKB1 promoter polymorphism was a useful molecular marker for the risk of recurrence in superficial bladder cancer. However, although most studies have analyzed the correlation of the $N F K B 1$ promoter polymorphism with tumors, ${ }^{31,34}$ those analyzing the effects of NFKB1 expression in osteosarcoma are limited. Thus, further experiments are needed to explore whether NFKB1 expression has any impact on osteosarcoma progression.

We also found that $\mathrm{E} 2$ treatment may repress the expression of genes, such as $R A R G, T B L 1 X$, and TCF7L2, involved in Wnt receptor signaling through $\beta$-catenin in U2OS osteosarcoma cells. The activation of Wnt signaling and the accumulation of $\beta$-catenin have been reported in many carcinomas, ${ }^{35,36}$ including osteosarcoma. ${ }^{37}$ TCF $7 L 2$ encodes the transcription factor TCF-4, which can be activated by dephosphorylated $\beta$-catenin via binding to a conserved $\mathrm{N}$-terminal region in the nucleus, thereby initiating the expression of target genes, including the proto-oncogenes $c$-jun and fra- $1 .{ }^{38}$ Thus, E2 treatment may have an unfavorable effect on U2OS osteosarcoma cells.

Among the other downregulated DEGs, $A K T 1$ was observed to have the highest degree in the PPI network. $A K T$, also known as protein kinase $\mathrm{B}$ (PKB; a serine/threonine kinase), is one of the most critical and versatile protein kinases involved in the mechanism of human physiology and disease. ${ }^{39,40}$ The activation of AKT pathways plays a central role in tumor metastasis. ${ }^{41}$ Furthermore, Fukaya et $\mathrm{al}^{42}$ have demonstrated the important role of AKT signaling in the pulmonary metastasis of osteosarcoma. The AKT family has three members: AKT1/PKB $\alpha$, AKT2/PKB $\beta$, and AKT3/PKB $\gamma$. $A K T 1$ and $A K T 2$ have been reported to be ubiquitously and similarly expressed in various tissues. ${ }^{43}$ Recently, Zhu et a ${ }^{44}$ have reported that elevated AKT2 expression is associated with poor outcomes in patients with osteosarcomas.

Here, $A K T 1$ was enriched in the cell cycle process, which was closely related to tumor progression. A study by Ju et $\mathrm{a}^{45}$ has revealed that $A K T 1$ governed breast cancer progression in mice, whereas another study has indicated that $A K T 1$ amplification regulates cisplatin (a chemotherapeutic agent) resistance in human lung cancer. ${ }^{46}$ Collectively, these reports have demonstrated that $A K T 1$ might be closely involved in osteosarcoma metastasis. In the current study, we predicted that $A K T 1$ expression was downregulated. According to qRTPCR results, although $A K T 1$ expression levels were increased when U2OS osteosarcoma cells were treated with E2, they were not significantly different. However, the effect of $A K T$ expression on U2OS osteosarcoma cells is controversial. For example, Nielsen-Preiss et $\mathrm{al}^{47}$ have reported that the downregulation of $A K T$ expression enhances osteosarcoma cell proliferation, whereas Díaz-Montero et $\mathrm{al}^{48}$ have found that $A K T$ expression is upregulated in anoikis-resistant human osteosarcoma SAOSar cells. Therefore, we speculated that E2 is involved in osteosarcoma metastasis, but the modulating mechanism is still unclear.

$A L C A M$ was another gene downregulated by E2 treatment in U2OS osteosarcoma cells, which was speculated to function in axonogenesis, cell morphogenesis involved in neuron differentiation, cell cycle processes, and neuron projection morphogenesis. ALCAM encodes the CD166 antigen, which is a $100-105 \mathrm{kDa}$ type-I transmembrane glycoprotein of the immunoglobulin protein superfamily. Similar to CD29, CD44,CD73, CD90, CD105, and CD106, it is known as a marker of the mesenchymal stem cell

Table 3 The top five GO terms of downregulated DEGs

\begin{tabular}{|c|c|c|c|c|}
\hline Category & Term & Description & $P$-value & Genes \\
\hline BP & GO:0060070 & Wnt receptor signaling pathway through $\beta$-catenin & 0.002951 & RARG, TBLIX, TCF7L2 \\
\hline $\mathrm{BP}$ & GO:0007409 & Axonogenesis & 0.01382 & ALCAM, NRPI, SLC26A6, etc \\
\hline $\mathrm{BP}$ & GO:0048667 & Cell morphogenesis involved in neuron differentiation & 0.01801 & ALCAM, NRPI, SLC26A6, etc \\
\hline $\mathrm{BP}$ & GO:0022402 & Cell cycle process & 0.01832 & AKTI, DSNI, POLDI, etc \\
\hline BP & GO:00488I2 & Neuron projection morphogenesis & 0.01916 & ALCAM, NRPI, SLC26A6, etc \\
\hline
\end{tabular}

Abbreviations: BP, biological process; GO, gene ontology; DEGs, differentially expressed genes. 


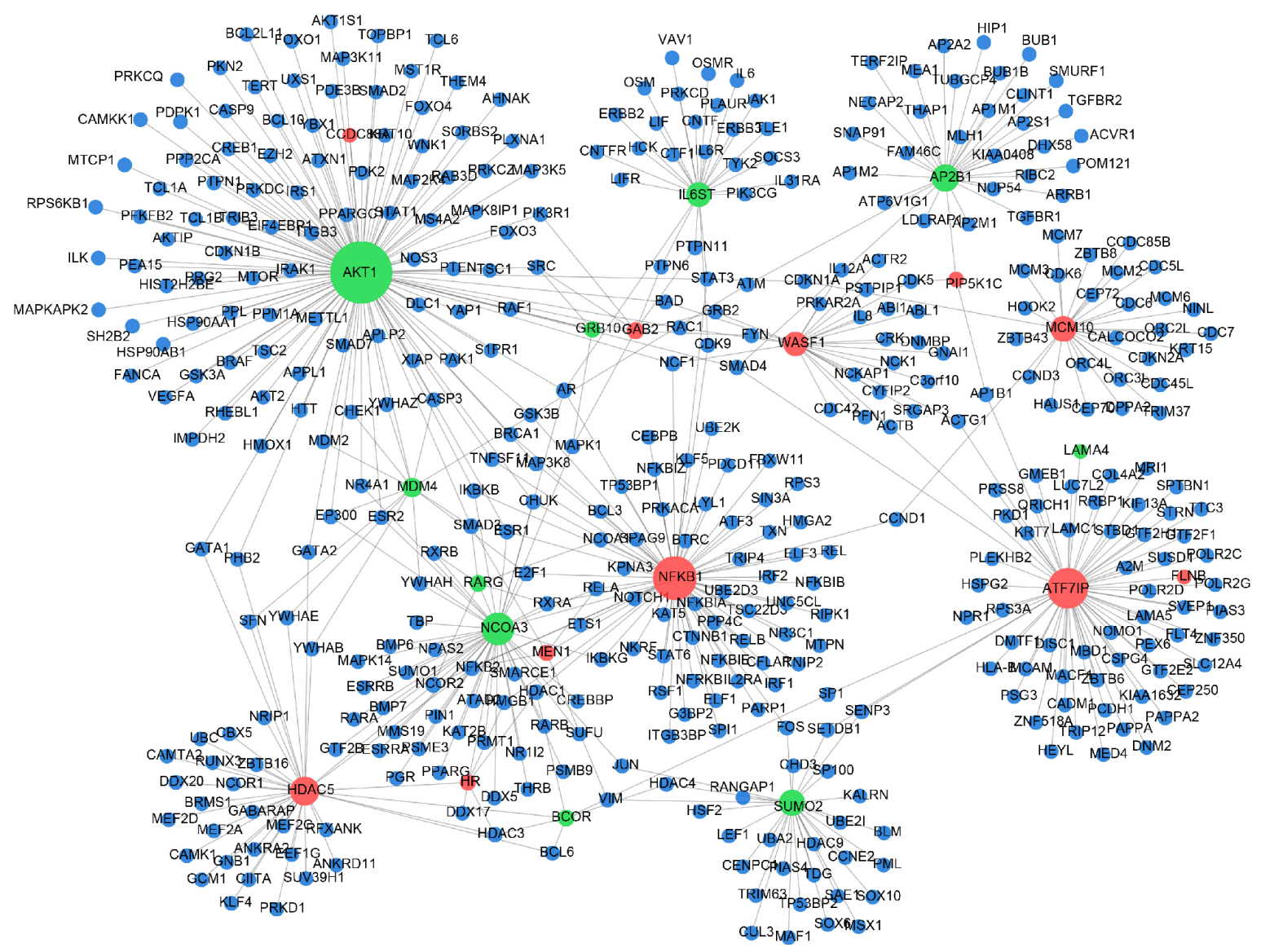

Figure I PPI network of DEGs.

Note: Red nodes represent upregulated DEGs, green nodes represent downregulated DEGs, and blue nodes represent non-DEGs.

Abbreviations: PPI, protein-protein interaction; DEGs, differentially expressed genes.
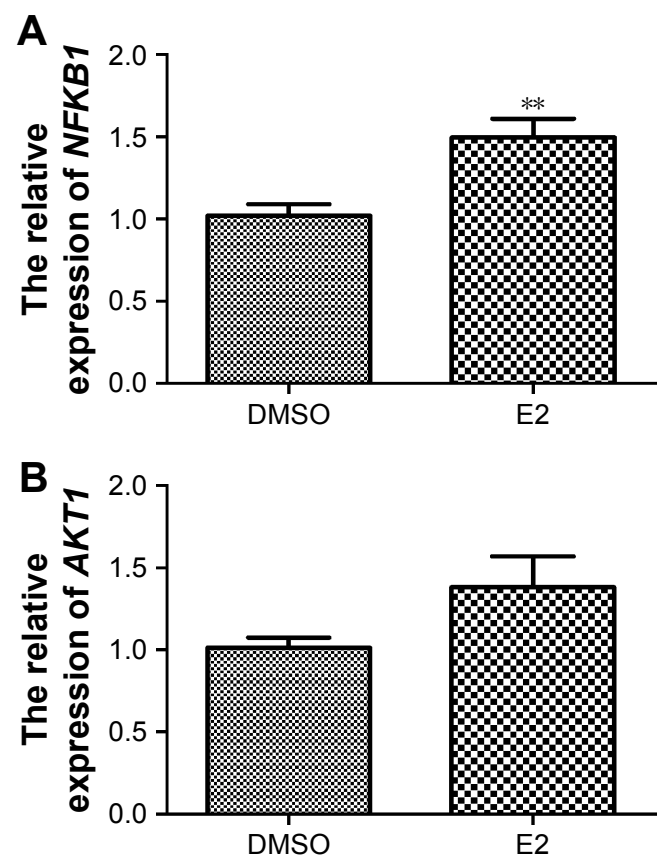

Figure 2 Related NFKBI (A) and AKTI (B) expression levels in U2OS osteosarcoma cells treated with E2.

Note: $* * P<0.01$.

Abbreviations: E2, estradiol; DMSO, dimethyl sulfoxide. phenotype. ${ }^{49,50}$ Its overexpression has also been reported in colorectal carcinoma ${ }^{51}$ and has been demonstrated to be associated with a poor prognosis of several tumors. ${ }^{51-53}$ Federman et al have reported that CD166 is highly expressed in the osteosarcoma cell lines HOS, KHOS, KHOS240s, and SJSA, but its expression status is not observed in U2OS osteosarcoma cells. ${ }^{54}$ They further proposed that this gene is a potential candidate for the targeted therapy of osteosarcoma. Our findings suggested that E2 treatment decreases ALCAM expression in U2OS osteosarcoma cells, thereby playing an inhibitory role against osteosarcoma.

Taken together, we found that E2 treatment may mainly upregulate the expression of genes, such as NFKB1, ATF7IP, and $H D A C 5$, related to the regulation of gene expression and transcription and downregulate that of genes involved in Wnt receptor signaling through $\beta$-catenin and morphogenesis (eg, TCF7L2, ALCAM, NRP1, SLC26A6, and AKT) in U2OS osteosarcoma cells. Thus, we proposed that E2 has an unfavorable effect against U2OS osteosarcoma cells. However, given that our findings were partly obtained using bioinformatics tools, they need to be further validated. 


\section{Acknowledgment}

This study was funded by a special fund for the medical service of the Jilin Finance Department (Grant No SCZSY201507).

\section{Disclosure}

The authors report no conflicts of interest in this work.

\section{References}

1. Ryan KJ. Biochemistry of aromatase: significance to female reproductive physiology. Cancer Res. 1982;42(8 suppl):3342s-3344s.

2. Hoppé E, Morel G, Biver E, et al. Male osteoporosis: do sex steroids really benefit bone health in men? Joint Bone Spine. 2011;78:S191-S196.

3. Yang X, Qin L, Liu J, Tian L, Qian H. 17 $\beta$-estradiol protects the liver against cold ischemia/reperfusion injury through the Akt kinase pathway. J Surg Res. 2012;178(2):996-1002.

4. Hartz AM, Mahringer A, Miller DS, Bauer B. 17- $\beta$-estradiol: a powerful modulator of blood-brain barrier BCRP activity. J Cereb Blood Flow Metab. 2010;30(10):1742-1755.

5. Mason HD, Willis DS, Beard RW, Winston RM, Margara R, Franks S. Estradiol production by granulosa cells of normal and polycystic ovaries: relationship to menstrual cycle history and concentrations of gonadotropins and sex steroids in follicular fluid. J Clin Endocrinol Metab. 1994;79(5):1355-1360.

6. Kelch R, Jenner M, Weinstein R, Kaplan S, Grumbach M. Estradiol and testosterone secretion by human, simian, and canine testes, in males with hypogonadism and in male pseudohermaphrodites with the feminizing testes syndrome. J Clin Invest. 1972;51(4):824.

7. Hess RA. Estrogen in the adult male reproductive tract: a review. Reprod Biol Endocrinol. 2003;1(1):52.

8. Barnabei VM, Cochrane BB, Aragaki AK, et al. Menopausal symptoms and treatment-related effects of estrogen and progestin in the women's health initiative. Obstet Gynecol. 2005;105(5, pt 1):1063-1073.

9. Warming L, Ravn P, Christiansen C. Levonorgestrel and 17 $\beta$-estradiol given transdermally for the prevention of postmenopausal osteoporosis. Maturitas. 2005;50(2):78-85.

10. Fink HA, Ewing SK, Ensrud KE, et al. Association of testosterone and estradiol deficiency with osteoporosis and rapid bone loss in older men. J Clin Endocrinol Metab. 2006;91(10):3908-3915.

11. Medina D. Mammary developmental fate and breast cancer risk. Endocr Relat Cancer. 2005;12(3):483-495.

12. Gunter MJ, Hoover DR, Yu H, et al. Insulin, insulin-like growth factor-I, endogenous estradiol, and risk of colorectal cancer in postmenopausal women. Cancer Res. 2008;68(1):329-337.

13. Tchafa AM, Zhong Z, Meng R, Quong JN, Quong AA. Increased cellular invasion and proliferation via estrogen receptor after $17-\beta$-estradiol treatment in breast cancer cells using stable isotopic labeling with amino acids in cell culture (SILAC). Adv Breast Cancer Res. 2013;2:32.

14. Tang N, Song W-X, Luo J, Haydon RC, He T-C. Osteosarcoma development and stem cell differentiation. Clin Orthop Relat Res. 2008; 466(9):2114-2130.

15. Marina N, Gebhardt M, Teot L, Gorlick R. Biology and therapeutic advances for pediatric osteosarcoma. Oncologist. 2004;9(4):422-441.

16. Zhou Q, Shen L, Liu C, Liu C, Chen H, Liu J. The effects of estradiol and glucocorticoid on human osteosarcoma cells: similarities and differences. Anticancer Res. 2016;36(4):1683-1691.

17. Kallio A, Guo T, Lamminen E, et al. Estrogen and the selective estrogen receptor modulator (SERM) protection against cell death in estrogen receptor alpha and beta expressing U2OS cells. Mol Cell Endocrinol. 2008;289(1):38-48.

18. Lima F, Vico L, Lafage-Proust MH, van der Saag P, Alexandre C, Thomas T. Interactions between estrogen and mechanical strain effects on $\mathrm{u} 2 \mathrm{os}$ human osteosarcoma cells are not influenced by estrogen receptor type. Bone. 2004;35(5):1127-1135.
19. Maran A, Zhang M, Kennedy A, et al. 2-methoxyestradiol induces interferon gene expression and apoptosis in osteosarcoma cells. Bone. 2002;30(2):393-398.

20. Góngora-Castillo E, Fedewa G, Yeo Y, Chappell J, DellaPenna D, Buell CR. Genomic approaches for interrogating the biochemistry of medicinal plant species. Methods Enzymol. 2012;517:139.

21. Trapnell C, Pachter L, Salzberg SL. TopHat: discovering splice junctions with RNA-seq. Bioinformatics. 2009;25(9):1105-1111.

22. Trapnell C, Roberts A, Goff L, et al. Differential gene and transcript expression analysis of RNA-seq experiments with TopHat and Cufflinks. Nat Protoc. 2012;7(3):562-578.

23. Trapnell C, Hendrickson DG, Sauvageau M, Goff L, Rinn JL, Pachter L. Differential analysis of gene regulation at transcript resolution with RNA-seq. Nat Biotechnol. 2013;31(1):46-53.

24. Huang da W, Sherman BT, Lempicki RA. Systematic and integrative analysis of large gene lists using DAVID bioinformatics resources. Nat Protoc. 2008;4(1):44-57.

25. Prasad TK, Goel R, Kandasamy K, et al. Human protein reference database - 2009 update. Nucleic Acids Res. 2009;37(suppl 1): D767-D772.

26. Saito R, Smoot ME, Ono K, et al. A travel guide to Cytoscape plugins. Nat Methods. 2012;9(11):1069-1076.

27. Newman ME. The structure and function of complex networks. SIAM Rev. 2003;45(2):167-256.

28. Aggarwal BB. Nuclear factor-kappaB: the enemy within. Cancer Cell. 2004;6(3):203-208.

29. Ota N, Nakajima T, Shirai Y, Emi M. Isolation and radiation hybrid mapping of a highly polymorphic CA repeat sequence at the human nuclear factor kappa-beta subunit 1 (NFKB1) locus. J Hum Genet. 1999;44(2):129-130.

30. Sakamoto K, Maeda S, Hikiba Y, et al. Constitutive NF-kappaB activation in colorectal carcinoma plays a key role in angiogenesis, promoting tumor growth. Clin Cancer Res. 2009;15(7):2248-2258.

31. Schumm K, Rocha S, Caamano J, Perkins ND. Regulation of p53 tumour suppressor target gene expression by the p52 NF-kappaB subunit. EMBO J. 2006;25(20):4820-4832.

32. Zhou B, Rao L, Li Y, et al. A functional insertion/deletion polymorphism in the promoter region of NFKB1 gene increases susceptibility for nasopharyngeal carcinoma. Cancer Lett. 2009;275(1):72-76.

33. Riemann K, Becker L, Struwe H, Rübben H, Eisenhardt A, Siffert W. Insertion/deletion polymorphism in the promoter of NFKB1 as a potential molecular marker for the risk of recurrence in superficial bladder cancer. Int J Clin Pharmacol Ther. 2007;45(8):423-430.

34. Lin SC, Liu CJ, Yeh WI, Lui MT, Chang KW, Chang CS. Functional polymorphism in NFKB1 promoter is related to the risks of oral squamous cell carcinoma occurring on older male areca (betel) chewers. Cancer Lett. 2006;243(1):47-54.

35. Lustig B, Behrens J. The WNT signaling pathway and its role in tumor development. J Cancer Res Clin Oncol. 2003;129(4):199-221.

36. Brabletz T, Jung A, Dag S, Hlubek F, Kirchner T. $\beta$-catenin regulates the expression of the matrix metalloproteinase-7 in human colorectal cancer. Am J Pathol. 1999;155(4):1033-1038.

37. Haydon RC, Deyrup A, Ishikawa A, et al. Cytoplasmic and/or nuclear accumulation of the $\beta$-catenin protein is a frequent event in human osteosarcoma. Int J Cancer. 2002;102(4):338-342.

38. Behrens J, von Kries JP, Kühl M, et al. Functional interaction of $\beta$-catenin with the transcription factor LEF-1. Nature. 1996;382(6592): 638-642.

39. Datta SR, Brunet A, Greenberg ME. Cellular survival: a play in three Akts. Genes Dev. 1999;13(22):2905-2927.

40. Manning BD, Cantley LC. AKT/PKB signaling: Navigating downstream. Cell. 2007;129(7):1261-1274.

41. Qiao M, Sheng S, Pardee AB. Metastasis and AKT activation. Cell Cycle. 2008;7(19):2991-2996.

42. Fukaya Y, Ishiguro N, Senga T, et al. A role for PI3K-AKT signaling in pulmonary metastatic nodule formation of the osteosarcoma cell line, LM8. Oncol Rep. 2005;14(4):847-852. 
43. Hanada M, Feng J, Hemmings BA. Structure, regulation and function of PKB/AKT - a major therapeutic target. Biochim Biophys Acta. 2004;1697(1):3-16.

44. Zhu Y, Zhou J, Ji Y, Yu B. Elevated expression of AKT2 correlates with disease severity and poor prognosis in human osteosarcoma. Mol Med Rep. 2014;10(2):737-742.

45. Ju X, Katiyar S, Wang C, et al. AKT1 governs breast cancer progression in vivo. Proc Natl Acad Sci U S A. 2007;104(18):7438-7443.

46. Liu LZ, Zhou XD, Qian G, Shi X, Fang J, Jiang BH. AKT1 amplification regulates cisplatin resistance in human lung cancer cells through the mammalian target of rapamycin/p70S6K1 pathway. Cancer Res. 2007;67(13):6325-6332.

47. Nielsen-Preiss SM, Silva SR, Gillette JM. Role of PTEN and Akt in the regulation of growth and apoptosis in human osteoblastic cells. J Cell Biochem. 2003;90(5):964-975.

48. Díaz-Montero CM, Wygant JN, Mcintyre BW. PI3-K/Akt-mediated anoikis resistance of human osteosarcoma cells requires Src activation. Eur J Cancer. 2006;42(10):1491.
49. Park B-W, Hah Y-S, Kim DR, Kim J-R, Byun J-H. Osteogenic phenotypes and mineralization of cultured human periosteal-derived cells. Arch Oral Biol. 2007;52(10):983-989.

50. Nadri S, Soleimani M, Kiani J, Atashi A, Izadpanah R. Multipotent mesenchymal stem cells from adult human eye conjunctiva stromal cells. Differentiation. 2008;76(3):223-231.

51. Weichert W, Knösel T, Bellach J, Dietel M, Kristiansen G. ALCAM/ CD166 is overexpressed in colorectal carcinoma and correlates with shortened patient survival. J Clin Pathol. 2004;57(11):1160-1164.

52. Tachezy M, Effenberger K, Zander H, et al. ALCAM (CD166) expression and serum levels are markers for poor survival of esophageal cancer patients. Int J Cancer. 2012;131(2):396-405.

53. Ishigami S, Ueno S, Arigami T, et al. Clinical implication of CD166 expression in gastric cancer. J Surg Oncol. 2011;103(1):57-61.

54. Federman N, Chan J, Nagy JO, et al. Enhanced growth inhibition of osteosarcoma by cytotoxic polymerized liposomal nanoparticles targeting the alcam cell surface receptor. Sarcoma. 2012:2012, 11 pages.
OncoTargets and Therapy

\section{Publish your work in this journal}

OncoTargets and Therapy is an international, peer-reviewed, open access journal focusing on the pathological basis of all cancers, potential targets for therapy and treatment protocols employed to improve the management of cancer patients. The journal also focuses on the impact of management programs and new therapeutic agents and protocols on

\section{Dovepress}

patient perspectives such as quality of life, adherence and satisfaction. The manuscript management system is completely online and includes a very quick and fair peer-review system, which is all easy to use. Visit http://www.dovepress.com/testimonials.php to read real quotes from published authors.

\footnotetext{
Submit your manuscript here: http://www.dovepress.com/oncotargets-and-therapy-journal
} 